\title{
Von Bill zum Bachelor
}

$I^{n}$ n diesen Tagen tritt die jüngste (nicht die letzte!) Gesundheitsreform in Kraft, und es gibt eigentlich niemand, der sie sich wirklich gewünscht hätte. Patienten nicht und auch nicht diejenigen, die ihre Leistungen mit den Krankenkassen abrechnen. Und auch die Krankenkassen nicht. Es musste sein, halten Gesundheitspolitiker dagegen, um „die Finanzierbarkeit unseres Gesundheitssystems zu erhalten“. Und irgendwie kommt es ja auch der Quadratur des Kreises nahe, einen auch nur halbwegs fairen Ausgleich der Interessen hinzubekommen, wenn einerseits die Kosten durch eine (vornehmlich demographisch bedingte) Zunahme des Bedarfs an medizinischen Leistungen stetig zunehmen, andererseits tendenziell immer weniger Menschen in den Solidartopf einbezahlen und diese nicht laufend stärker belastet werden können. Nicht immer ergreift die Politik die Initiative, weil es gilt, eine offensichtlich herannahende Katastrophe abzuwenden (oder sich zumindest wieder eine Verschnaufpause zu schaffen). Manchmal steigt der Druck zum Handeln auch aus anderen Gründen, z. B. wenn die Rechtssprechung einen gesetzlich geregelten Aspekt weiter entwickelt, bis das Gesetz nicht mehr so recht passt. Das könnte vielleicht schon bald in der Frage der Ausübung der Heilkunde durch nichtärztliche medizinische Fachberufe der Fall sein. Wenn, dann hat das sog. „Bill-Urteil“ des Oberverwaltungsgerichts Rheinland-Pfalz vom 21.11.06 dazu einen gehörigen Beitrag geleistet.

In diesem Verfahren hatten die Kläger, unter ihnen auch Physiotherapeuten, die Erlaubnis zur Ausübung der Heilkunde ohne Eignungsprüfung beantragt, allerdings beschränkt auf den in $\S 3$ und 8 des Gesetzes zur Regelung der Berufe in der Physiotherapie beschriebenen Bereich der Physiotherapie und physikalischen Therapie. Das Gericht stellte nämlich fest, dass die genannten Tätigkeiten zwar grundsätzlich der Heilpraktikererlaubnis bedürfen, einem staatlich geprüften Physiotherapeuten diese „gegenständlich beschränkte Heilpraktikererlaubnis“ jedoch ohne weitere Eignungsprüfung erteilt werden muss. Schließlich gäbe es, so das Gericht, keinen „anerkennenswerten Grund“ dafür, „von einem staatlich geprüften Physiotherapeuten, der erklärtermaßen nicht außerhalb seines Fachgebiets behandeln möchte“, zu verlangen, eine Befähigungsprüfung nach dem Heilpraktikergesetz zu absolvieren.

Natürlich gilt das Urteil erst einmal nur in Rheinland-Pfalz, es ist aber zu erwarten, dass ähnliche Urteile zu anderen Klagen hinzukommen. Zum Beispiel bei solchen von Therapeuten aus anderen EU-Ländern. Oder auch von Patienten, die bestimmte Behandlungen nach Inkrafttreten der Gesundheitsreform aus eigener Tasche bezahlen müssen, und sich daher nicht länger gängeln lassen wollen, bei irgendeiner Arztpraxis nach unkalkulierbarer Wartezeit eine lieblose Überweisung abzuholen, bevor sie sich dem Therapeuten ihres Vertrauens anvertrauen.

Der so genannte Bologna-Prozess, eine schon 1999 von den Wissenschaftsministern der EU gemeinsam beschlossene Vereinheitlichung des Systems der akademischen Ausbildung in den Ländern der EU, wird zwangsläufig zu einer Akademisierung fundierter medizinischer Fachberufe führen. Für die Osteopathie eine hervorragende Chance, über die Akademisierung auch die „staatliche Anerkennung“ zu erreichen, für praktizierende Osteopathen die Perspektive, mit dem Abschluss eines Aufbaustudiums zum „Bachelor“ sich perspektivisch die „Lizenz“ zur freien Berufsausübung zu sichern. Der VOD bemüht sich deshalb auch intensiv um diese Institutionalisierung.

Die Herausgeber

Skelett- und Weichteilelemente eines rechten Ellenbogengelenks. Aus: Schünke M, Schulte E, Schumacher U, Prometheus LernAtlas der Anatomie. Allgemeine Anatomie und Bewegungssystem. Illustrationen von Voll M und Wesker K. Stuttgart: Thieme; 2005. Bearbeitung: Pdesign A Page. Stuttgart 2007 